\title{
AN ALMA CONTINUUM SURVEY OF CIRCUMSTELLAR DISKS IN THE UPPER SCORPIUS OB ASSOCIATION
}

\author{
John M. CARPenter, LuCA Ricci, And Andrea IsElla \\ Department of Astronomy, California Institute of Technology, MC 249-17, Pasadena, CA 91125, USA \\ Received 2013 December 31; accepted 2014 March 28; published 2014 May 2
}

\begin{abstract}
We present ALMA $880 \mu \mathrm{m}$ continuum observations of $20 \mathrm{~K}$ - and M-type stars in the Upper Scorpius OB association (Upper Sco) that are surrounded by protoplanetary disks. These data are used to measure the dust content in disks around low-mass stars $\left(0.1-1.6 M_{\odot}\right)$ at a stellar age of 5-11 Myr. Thirteen sources were detected in the $880 \mu \mathrm{m}$ dust continuum at $\geqslant 3 \sigma$ with inferred dust masses between 0.3 and $52 M_{\oplus}$. The dust masses tend to be higher around the more massive stars, but the significance is marginal in that the probability of no correlation is $p \approx 0.03$. The evolution in the dust content in disks was assessed by comparing the Upper Sco observations with published continuum measurements of disks around $\sim 1-2$ Myr stars in the Class II stage in the Taurus molecular cloud. While the dust masses in the Upper Sco disks are on average lower than in Taurus, any difference in the dust mass distributions is significant at less than $3 \sigma$. For stellar masses between $0.49 M_{\odot}$ and $1.6 M_{\odot}$, the mean dust mass in disks is lower in Upper Sco relative to Taurus by $\Delta \log M_{\text {dust }}=0.44 \pm 0.26$.
\end{abstract}

Key words: open clusters and associations: individual (Upper Scorpius OB1) - protoplanetary disks stars: pre-main sequence

\section{INTRODUCTION}

The lifetime of optically thick, gas-rich disks surrounding young stars provides empirical constraints on the timescales to form planetary systems and the mechanisms responsible for disk dispersal. The disk dissipation timescale is typically measured by surveying clusters or associations of stars of various ages and identifying the fraction of stars that exhibit infrared emission in excess of the stellar photosphere, which is attributed to a circumstellar disk that absorbs and re-radiates the stellar radiation. Infrared surveys have shown that $\sim 80 \%$ of $\mathrm{K}$ - and M-type stars are surrounded by a disk at an age of $\sim 1 \mathrm{Myr}$ and decline to $\lesssim 20 \%$ at an age of $\sim 5 \mathrm{Myr}$ (Haisch et al. 2001; Mamajek et al. 2004; Hernández et al. 2008). Disks around A- and B-type stars $\left(\sim 2-3 M_{\odot}\right)$ appear to evolve on even shorter timescales (Hernández et al. 2005; Carpenter et al. 2006; Dahm \& Carpenter 2009).

Submillimeter continuum observations provide additional key diagnostics of disk evolution. Whereas infrared emission is generally optically thick and traces the disk surface layer within $\sim 1 \mathrm{AU}$ of the star, submillimeter continuum emission is optically thin over most of the disk and can also probe the cool, outer disk. The submillimeter continuum emission is a measure of the surface area of millimeter-sized particles in the disk (e.g., Ricci et al. 2010b) and can be used to estimate the dust mass with assumptions on the dust opacity and temperature structure of the disk.

Hundreds of $\sim 1-2$ Myr old stars in the Taurus and Ophiuchus clouds have been surveyed in the submillimeter continuum with single-dish telescopes and interferometers (Beckwith et al. 1990; André \& Montmerle 1994; Motte et al. 1998; Andrews \& Williams 2005, 2007b; Schaefer et al. 2009; Andrews et al. 2013), and the continuum and/or spectral-line emission have been resolved in dozens of stars (Dutrey et al. 1996; Simon et al. 2000; Kitamura et al. 2002; Andrews \& Williams 2007a; Isella et al. 2009; Andrews et al. 2009, 2010; Kwon et al. 2011; Guilloteau et al. 2011). Collectively, these extensive observations have established the disk properties around lowmass stars at an age of $\sim 1-2 \mathrm{Myr}$.
Submillimeter observations of stars at other ages are more limited; nonetheless, they have begun to reveal how the dust mass evolves. Submillimeter and millimeter observations of the 2-3 Myr IC 348 (Carpenter 2002; Lee et al. 2011) and the 5-11 Myr Upper Scorpius OB association (hereafter Upper Sco; Mathews et al. 2012b) demonstrate that these regions lack the luminous disks found in Taurus and Ophiuchus. However, the stellar samples observed so far in IC 348 and Upper Sco are incomplete, and Andrews et al. (2013) have suggested that the lack of bright disks may be due to a selection bias toward latetype stars rather than to an intrinsically different distribution of disk submillimeter luminosities. After considering the lower mean submillimeter flux density observed in disks around lowermass stars, Andrews et al. (2013) showed that the millimeterwavelength luminosity distribution of the IC 348 and Taurus samples are statistically indistinguishable, while the Upper Sco sample appears to have only marginally $(\sim 2.5 \sigma)$ lower luminosities on average.

More recently, Williams et al. (2013) presented a large submillimeter survey of disks in the $\sim 3$ Myr old $\sigma$ Orionis cluster. In this case, they found that the submillimeter luminosities are lower in $\sigma$ Orionis than in Taurus, indicating a decline of the amount of material in disks as star-forming regions age from $\sim 1$ to $\sim 3 \mathrm{Myr}$.

We report new submillimeter continuum observations of K- and M-type stars in Upper Sco obtained with ALMA during Cycle 0 Early Science. These data achieve nearly an order of magnitude better sensitivity than previous submillimeter surveys of disks in Upper Sco. We use these data to investigate any dependence of the disk properties with stellar mass and compare these observations with existing submillimeter continuum measurements of stars in the younger Taurus region to investigate the evolution of dust masses.

\section{THE UPPER SCO SAMPLE}

The initial sample consisted of $24 \mathrm{~K}$ - and M-type stars in Upper Sco that were identified with an infrared excess between $3.6 \mu \mathrm{m}$ and $16 \mu \mathrm{m}$ by Carpenter et al. (2006). The characteristics 
Table 1

Observed Sources

\begin{tabular}{lccc}
\hline \hline Source & \multicolumn{2}{c}{ Phase Center (J2000) } & \multirow{2}{*}{ UT Date Observed } \\
\cline { 2 - 3 } & Right Ascension & Declination & \\
\hline [PBB2002] J155624.8-222555 & $15: 56: 24.774$ & $-22: 25: 55.26$ & 2012 Aug 24 \\
[PBB2002] J155706.4-220606 & $15: 57: 06.419$ & $-22: 06: 06.10$ & 2012 Aug 24 \\
[PBB2002] J155729.9-225843 & $15: 57: 29.862$ & $-22: 58: 43.85$ & 2012 Aug 24 \\
[PBB2002] J155829.8-231007 & $15: 58: 29.813$ & $-23: 10: 07.72$ & 2012 Aug 24 \\
[PZ99] J160357.6-203105 & $16: 03: 57.677$ & $-20: 31: 05.51$ & 2012 Dec 16 \\
[PZ99] J160421.7-213028 & $16: 04: 21.655$ & $-21: 30: 28.40$ & 2012 Aug 27 \\
[PBB2002] J160525.5-203539 & $16: 05: 25.564$ & $-20: 35: 39.71$ & 2012 Aug 24 \\
[PBB2002] J160532.1-193315 & $16: 05: 32.152$ & $-19: 33: 15.99$ & 2012 Aug 24 \\
[PBB2002] J160600.6-195711 & $16: 06: 00.616$ & $-19: 57: 11.46$ & 2012 Aug 27 \\
ScoPMS 31 & $16: 06: 21.963$ & $-19: 28: 44.56$ & 2012 Dec 16 \\
[PBB2002] J160622.8-201124 & $16: 06: 22.781$ & $-20: 11: 24.28$ & 2012 Aug 27 \\
[PBB2002] J160643.8-190805 & $16: 06: 43.860$ & $-19: 08: 05.56$ & 2012 Dec 16 \\
[PBB2002] J160702.1-201938 & $16: 07: 02.118$ & $-20: 19: 38.77$ & 2012 Aug 24 \\
[PBB2002] J160823.2-193001 & $16: 08: 23.245$ & $-19: 30: 00.95$ & 2012 Dec 16 \\
[PBB2002] J160827.5-194904 & $16: 08: 27.520$ & $-19: 49: 04.72$ & 2012 Aug 27 \\
[PBB2002] J160900.0-190836 & $16: 09: 00.020$ & $-19: 08: 36.80$ & 2012 Aug 27 \\
[PBB2002] J160900.7-190852 & $16: 09: 00.761$ & $-19: 08: 52.68$ & 2012 Dec 16 \\
[PBB2002] J160959.4-180009 & $16: 09: 59.341$ & $-18: 00: 09.08$ & 2012 Aug 24 \\
[PZ99] J161411.0-230536 & $16: 14: 11.077$ & $-23: 05: 36.24$ & 2012 Aug 27 \\
[PBB2002] J161420.2-190648 & $16: 14: 20.299$ & $-19: 06: 48.14$ & 2012 Aug 27 \\
\hline
\end{tabular}

of the infrared excess suggest that these stars are surrounded by optically thick disks in the Class II evolutionary stage (Lada \& Wilking 1984). Table 1 lists the 20 sources observed with ALMA, the phase center of the ALMA observations, and the date of the ALMA observations. The four sources that were not observed before the end of Cycle 0 are J161115.3-175721 (M1 spectral type), J160545.4-202308 (M2), J160357.9-194210 (M2), and J160953.6-175446 (M3).

The stellar luminosity $\left(L_{*}\right)$, effective temperature $\left(T_{*}\right)$, and mass $\left(M_{*}\right)$ were estimated based on available photometry and spectroscopy. The effective temperature scale was set based on the observed spectral type. In anticipation of comparing the ALMA observations of Upper Sco with observations of Taurus presented in the literature, the temperature scale described in Andrews et al. (2013, and references therein) was adopted.

Observed optical and near-infrared photometry were drawn from the Two Micron All Sky Survey (Skrutskie et al. 2006; Cutri et al. 2003) and DENIS (The DENIS Consortium 2005) photometric catalogs. The visual extinction was estimated from the observed DENIS $I-J$ color by adopting the intrinsic colors for 5-30 Myr stars from Pecaut \& Mamajek (2013) and the Cardelli et al. (1989) extinction law. DENIS photometry was not available for three sources, for which we adopt $A_{V}=0.7 \mathrm{mag}$ (the median value for the remaining stars) and an uncertainty of $0.5 \mathrm{mag}$. For J161420.2-190648, the visual extinction derived from the DENIS $I-J$ color $\left(A_{V}=4.0 \pm 0.27 \mathrm{mag}\right)$ produced a visual extinction significantly larger than derived by Preibisch et al. (2002) from $R-I$ photometry $\left(A_{V}=1.8\right)$. This star exhibits excess emission in the near-infrared bands that may contribute to the $J$-band photometry (Dahm \& Carpenter 2009); we adopted $A_{V}=2 \pm 0.5 \mathrm{mag}$ for this star.

The uncertainties in the effective temperatures assume a spectral-type uncertainty of \pm 1 subclass. The uncertainties in the luminosities include the uncertainty in the $J$-band photometry, the extinction, and the distance to Upper Sco, which is assumed to be $15 \%$ of the mean distance of $145 \mathrm{pc}$ (de Zeeuw et al. 1999). Assuming Gaussian distributions in $\log L_{*}$ and $\log T_{*}$, the distribution of possible stellar masses (and ages) were derived using the Siess et al. (2000) pre-main-sequence evolutionary tracks with a metallicity of $Z=0.02$ and no convective overshoot. The stellar mass distribution was then inferred by marginalizing over the stellar ages. Table 2 lists the derived stellar parameters.

\section{ALMA OBSERVATIONS}

The ALMA Early Science Cycle 0 observations were obtained on 2012 August 24 UT (seven sources), 2012 August 28 (six sources), and 2012 December 16 (five sources). Table 3 summarizes the observations, including the number of $12 \mathrm{~m}$ antennas used, the minimum and maximum project baselines, the primary flux calibrator, a secondary flux calibrator, the passband calibrator, and the gain calibrator for each day.

All observations were obtained in band 7 with a FWHM primary beam size of 18 .'5. The correlator was configured to record dual polarization for spectral windows centered on 333.8, $335.7,345.8$, and $347.7 \mathrm{GHz}$ for a mean frequency of $340.7 \mathrm{GHz}$ $(880 \mu \mathrm{m})$. Each window provided a bandwidth of $1.875 \mathrm{GHz}$ with channel widths of $0.488 \mathrm{MHz}$. The spectral resolution is twice the channel width. One spectral window was centered on the ${ }^{12} \mathrm{CO} J=3-2$ line at a rest frequency of $345.79599 \mathrm{GHz}$. The channels with ${ }^{12} \mathrm{CO} J=3-2$ emission were omitted when analyzing the continuum data.

The ALMA data were calibrated using the CASA package. The initial reduction scripts were kindly provided by Crystal Brogan (NRAO), which included phase calibration with the $183 \mathrm{GHz}$ water vapor radiometers, bandpass calibration, flux calibration, and gain calibration. Table 3 lists the calibrators for each night. We adapted the initial calibration scripts to perform bandpass, flux, and gain calibration using CASA 4.1.

Flux calibration was established by observing either Neptune or Titan and adopting the Butler-JPL-Horizon 2012 models. Due to the broad ${ }^{12} \mathrm{CO} J=3-2$ absorption line present in the atmospheres of Neptune and Titan at $345.8 \mathrm{GHz}$, we measured the flux densities of the bandpass and gain calibrators only in the 333.8 GHz and 335.7 GHz spectral windows. The flux densities measured in these two windows were consistent to within $3 \%$ 
Table 2

Stellar Properties

\begin{tabular}{|c|c|c|c|c|c|}
\hline Source & $\mathrm{SpT}$ & $A_{V}$ & $\log \left(T_{*} / K\right)$ & $\log \left(L_{*} / L_{\odot}\right)$ & $\log \left(M_{*} / M_{\odot}\right)$ \\
\hline [PBB2002] J155624.8-222555 & M4 & $0.69 \pm 0.36$ & $3.514 \pm 0.019$ & $-1.18 \pm 0.14$ & $-0.66(-0.14,+0.10)$ \\
\hline [PBB2002] J155706.4-220606 & M4 & $0.70 \pm 0.50^{\mathrm{a}}$ & $3.514 \pm 0.019$ & $-1.44 \pm 0.14$ & $-0.68(-0.18,+0.09)$ \\
\hline [PBB2002] J155729.9-225843 & M4 & $0.70 \pm 0.50^{\mathrm{a}}$ & $3.514 \pm 0.019$ & $-1.33 \pm 0.14$ & $-0.69(-0.14,+0.12)$ \\
\hline [PBB2002] J155829.8-231007 & M3 & $1.07 \pm 0.40$ & $3.533 \pm 0.018$ & $-1.31 \pm 0.14$ & $-0.58(-0.10,+0.12)$ \\
\hline [PZ99] J160357.6-203105 & K5 & $0.70 \pm 0.50^{\mathrm{a}}$ & $3.638 \pm 0.024$ & $-0.17 \pm 0.14$ & $0.01(-0.05,+0.08)$ \\
\hline [PZ99] J160421.7-213028 & $\mathrm{K} 2$ & $0.66 \pm 0.27$ & $3.690 \pm 0.016$ & $-0.24 \pm 0.14$ & $-0.02(-0.04,+0.05)$ \\
\hline [PBB2002] J160525.5-203539 & M5 & $0.37 \pm 0.41$ & $3.495 \pm 0.020$ & $-1.37 \pm 0.14$ & $-0.88(-0.15,+0.17)$ \\
\hline [PBB2002] J160532.1-193315 & M5 & $0.19 \pm 0.42$ & $3.495 \pm 0.020$ & $-1.59 \pm 0.14$ & $-1.01(-0.06,+0.25)$ \\
\hline [PBB2002] J160600.6-195711 & M5 & $0.22 \pm 0.37$ & $3.495 \pm 0.020$ & $-1.20 \pm 0.14$ & $-0.79(-0.18,+0.11)$ \\
\hline ScoPMS 31 & M0.5 & $0.98 \pm 0.26$ & $3.577 \pm 0.020$ & $-0.28 \pm 0.14$ & $-0.31(-0.09,+0.12)$ \\
\hline [PBB2002] J160622.8-201124 & M5 & $0.00 \pm 0.37$ & $3.495 \pm 0.020$ & $-1.39 \pm 0.14$ & $-0.89(-0.17,+0.15)$ \\
\hline [PBB2002] J160643.8-190805 & K6 & $0.72 \pm 0.25$ & $3.624 \pm 0.015$ & $-0.39 \pm 0.14$ & $-0.04(-0.05,+0.04)$ \\
\hline [PBB2002] J160702.1-201938 & M5 & $0.92 \pm 0.37$ & $3.495 \pm 0.020$ & $-1.49 \pm 0.14$ & $-0.90(-0.17,+0.15)$ \\
\hline [PBB2002] J160823.2-193001 & K9 & $1.05 \pm 0.29$ & $3.593 \pm 0.023$ & $-0.55 \pm 0.14$ & $-0.13(-0.16,+0.07)$ \\
\hline [PBB2002] J160827.5-194904 & M5 & $0.70 \pm 0.39$ & $3.495 \pm 0.020$ & $-1.16 \pm 0.14$ & $-0.78(-0.18,+0.12)$ \\
\hline [PBB2002] J160900.0-190836 & M5 & $0.42 \pm 0.35$ & $3.495 \pm 0.020$ & $-1.32 \pm 0.14$ & $-0.84(-0.20,+0.15)$ \\
\hline [PBB2002] J160900.7-190852 & K9 & $1.32 \pm 0.25$ & $3.593 \pm 0.023$ & $-0.38 \pm 0.14$ & $-0.08(-0.23,+0.04)$ \\
\hline [PBB2002] J160959.4-180009 & M4 & $0.56 \pm 0.36$ & $3.514 \pm 0.019$ & $-1.00 \pm 0.14$ & $-0.59(-0.17,+0.07)$ \\
\hline [PZ99] J161411.0-230536 & $\mathrm{K} 2$ & $0.48 \pm 0.25$ & $3.690 \pm 0.030$ & $0.41 \pm 0.14$ & $0.20(-0.07,+0.05)$ \\
\hline [PBB2002] J161420.2-190648 & K5 & $2.00 \pm 0.50^{\mathrm{a}}$ & $3.638 \pm 0.024$ & $-0.29 \pm 0.14$ & $0.01(-0.07,+0.04)$ \\
\hline
\end{tabular}

Note. ${ }^{\text {a }}$ Photometry is not available to derive $A_{V}$; the assumed value is listed (see the text).

Table 3

ALMA Observations

\begin{tabular}{lccccccc}
\hline \hline UT Date & \multirow{2}{*}{$\begin{array}{c}\text { Number } \\
\text { Antennas }\end{array}$} & Baseline Range & pwv & \multicolumn{3}{c}{ Calibrators } \\
\cline { 5 - 8 } & $(\mathrm{m})$ & $(\mathrm{mm})$ & Flux & Passband & Secondary & Gain \\
\hline 2012 Aug 24 & 25 & $17-375$ & 0.77 & Neptune & J1924-292 & J1751-0939 & J1625-2527 \\
2012 Aug 28 & 28 & $12-386$ & 0.68 & Titan & J1924-292 & J1751-0939 & J1625-2527 \\
2012 Dec 16 & 17 & $16-402$ & 1.16 & Titan & J1924-292 & $\ldots$ & J1625-2527 \\
\hline
\end{tabular}

for a given source on a single day. The average flux density in these two windows was adopted for all four spectral windows.

The measured flux densities of the passband, secondary, and gain calibrators were $16 \%$ brighter on average for the 2012 August 28 data than on 2012 August 24. Given the measurements were obtained four days apart and the flux differences were common to three different calibrators, we assume that this represents a systematic difference in the absolute flux calibration between the two data sets. For these two days, we averaged the two flux measurements for each calibrator. The adopted flux density for the gain calibrator J1625-2527 on these two nights was $0.97 \mathrm{Jy}$. We adopt a $1 \sigma$ calibration uncertainty of $10 \%$.

Images were created from the calibrated visibilities using CASA 4.1 with a Briggs robust weighting parameter of two. A continuum map was produced by averaging all of the channels except those around the ${ }^{12} \mathrm{CO} J=3-2$ line. The $1 \sigma$ pointsource sensitivity near the phase center is typically $0.19,0.16$, and $0.52 \mathrm{mJy} \mathrm{beam}^{-1}$ for sources observed on 2012 August 24, 2012 August 28, and 2012 December 16, respectively.

\section{ALMA RESULTS}

Figure 1 presents contour maps on the $880 \mu \mathrm{m}$ continuum emission for the Upper Sco sample. Each image is centered on the expected stellar position, which was computed using the coordinates and proper motions in the PPMXL catalog (Roeser et al. 2010). The median offset of the expected stellar position from the phase center of the ALMA observations is $(\Delta \alpha, \Delta \delta)=\left(-0{ }^{\prime \prime} 17,-0{ }^{\prime} 33\right)$.
Figure 2 presents the real component of the observed continuum visibilities for each source. The continuum emission toward four sources (J160421.7-213028, J160823.2-193001, $\mathrm{J} 160900.7-190852$, and J161420.2-190648) are clearly resolved in that the visibilities decline in amplitude with increasing $u v$ distance. The four resolved sources are also the brightest disks in the sample with flux densities in excess of $40 \mathrm{mJy}$ at $880 \mu \mathrm{m}$; the remaining sources have flux densities less than $6 \mathrm{mJy}$. The dust emission around J160421.7-213028 is resolved into a ring, which was previously imaged in the $880 \mu \mathrm{m}$ continuum with the Submillimeter Array (Mathews et al. 2012a) and scattered light (Mayama et al. 2012). Zhang et al. (2014) present an extensive analysis of the ALMA continuum and molecular line data for this source. Model fitting for the remaining sources and along with analysis of the ${ }^{12} \mathrm{CO} J=3-2$ data will be presented in a separate paper.

Flux densities were measured by fitting an elliptical Gaussian to the visibility data using uvmodelfit in CASA. The model contains six free parameters: (1) the integrated flux density, (2) the FWHM, (3) the aspect ratio, (4) the position angle, (5) the right ascension offset from the phase center, and (6) the declination offset from the phase center. The uncertainty on the model parameters were scaled by the factor needed to produce a reduced chi-squared of unity. If the ratio of the FWHM to the uncertainty in the FWHM was less than two, a pointsource model with three free parameters (integrated intensity and position offsets) was fitted to the visibility data instead. For the disk ring around J160421.7-213028, the flux density was measured using aperture photometry in a circular aperture of radius 1 1.5 in the deconvolved image. 


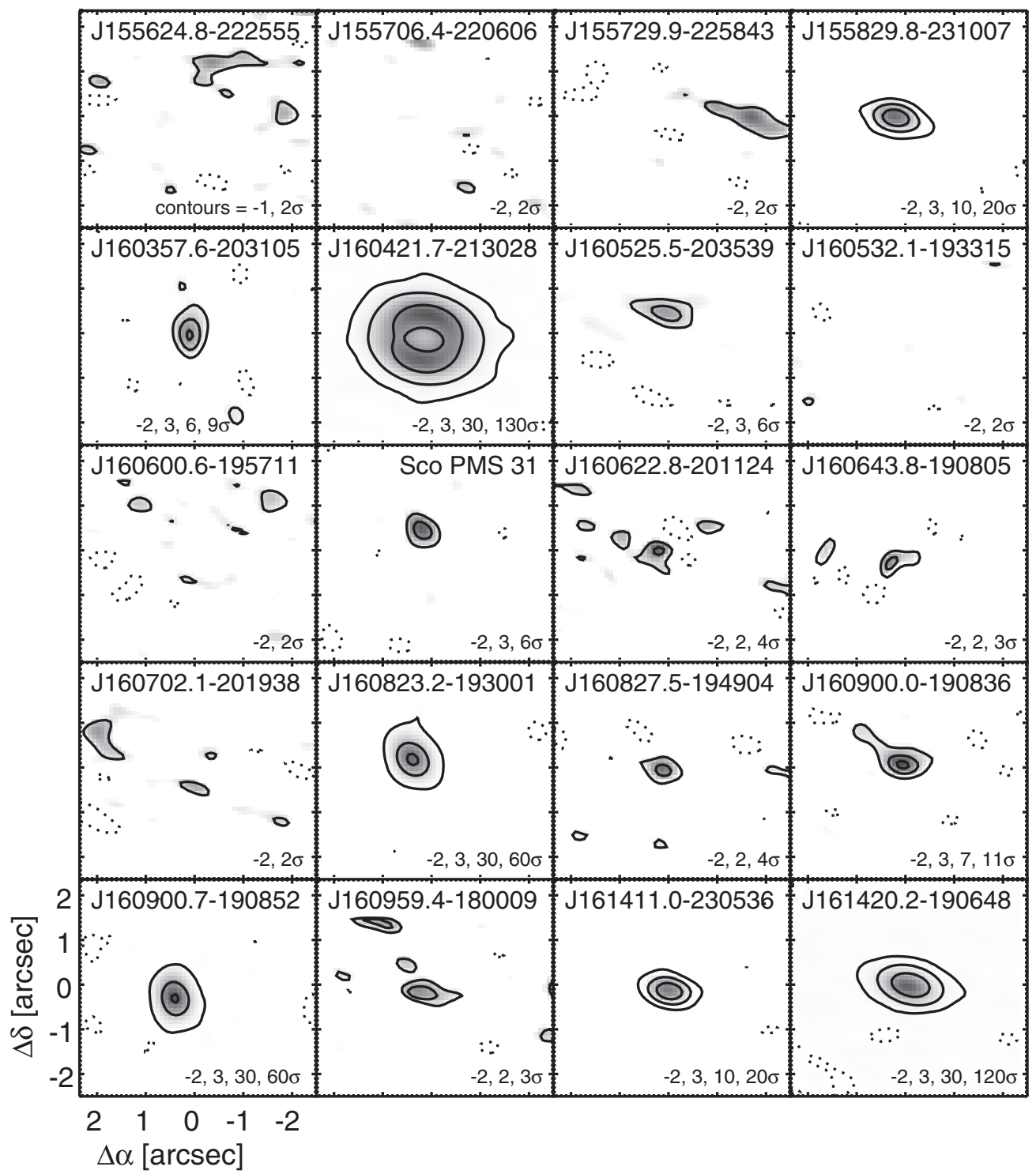

Figure 1. Contour maps of the ALMA $880 \mu$ m continuum emission for $20 \mathrm{~K}-\mathrm{M}$ type stars in Upper Sco. Each map is centered on the stellar position after correction for proper motion. The contour levels are indicated in the lower right of each panel, where solid and dotted contours indicate positive and negative flux densities, respectively.

Table 4 summarizes the continuum measurements. The table includes the integrated flux density, the offset of the submillimeter emission from the stellar position, the rms noise in the synthesized image, and the FWHM and position angle of the deconvolved beam. The uncertainty in the offsets include the uncertainties in the stellar position at the measured epoch in the PPMXL catalog, the proper motion propagated since that epoch, and the centroid of the submillimeter continuum emission. Upper limits to the flux density were computed as $\max \left(0, S_{v}\right)+$ $3 \times$ rms, assuming that the emission originates from a point source.

Thirteen sources were detected in the $880 \mu \mathrm{m}$ continuum with a signal-to-noise ratio of 3 or greater. Within the $3 \sigma$ uncertainties, the centroid of the continuum emission is consistent with the stellar position, with a median offset between the centroid of the $880 \mu \mathrm{m}$ continuum and the expected stellar position of $(\Delta \alpha, \Delta \delta)=\left(0^{\prime} .02,0^{\prime} .05\right)$. We conclude that most of the ALMA detections must be associated with the star and that there are no clear examples of extragalactic contamination in the sample.
We assume throughout this paper that the detected continuum sources are associated with the Upper Sco stars.

\section{PROPERTIES OF DISKS IN UPPER SCO}

In this section, the ALMA continuum measurements are used to infer the mass of dust in the circumstellar disks. After describing how the dust masses are estimated, we examine if the dust masses vary systematically with stellar mass within Upper Sco. We then compare the dust mass distribution with stars in the younger Taurus molecular cloud to constrain the evolution of dust mass with time.

\subsection{Dust Masses}

Interferometric observations can be used to measure the dust masses in disks by fitting a parameterized surface density model to the observed visibilities. While interferometric data are available for the entire Upper Sco sample, such data are not available for all sources in the Taurus comparison sample 

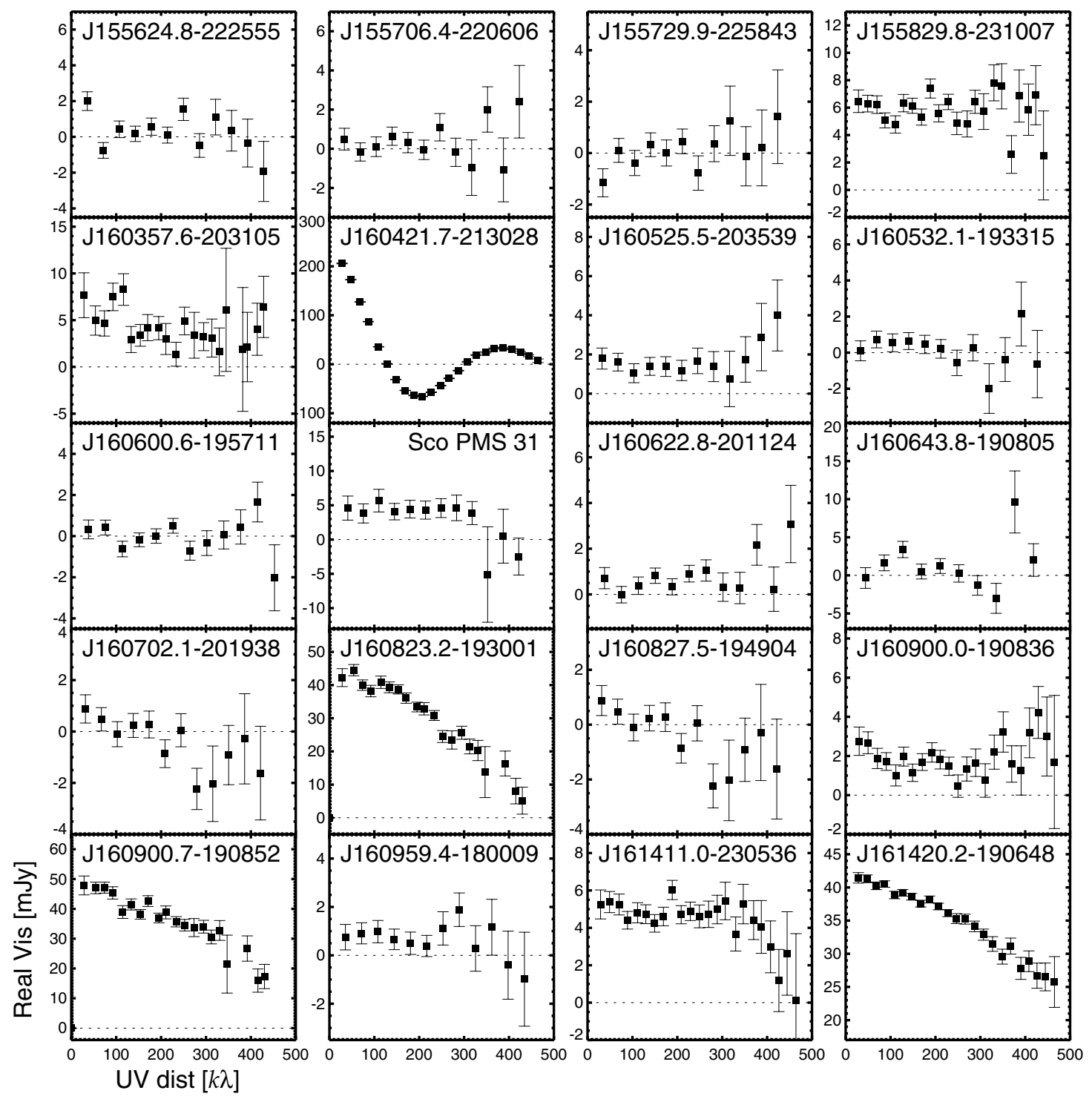

Figure 2. Real part of the visibility as a function of projected baseline length for the ALMA $880 \mu \mathrm{m}$ continuum data for $20 \mathrm{~K}-\mathrm{M}$ type stars in Upper Sco. The phase center has been shifted to correspond to the centroid of the continuum emission, or the stellar position if the continuum is not detected.

described below. Therefore, we adopt a simplified approach to estimating dust masses that can be applied to all sources, as outlined in Andrews et al. (2013). Assuming the dust emission is isothermal and optically thin, the dust mass is given by

$$
\log M_{\text {dust }}=\log S_{v}+2 \log d-\log \kappa_{v}-\log B_{v}\left(T_{\text {dust }}\right),
$$

where $S_{v}$ is the observed flux density, $d$ is the distance, $\kappa_{v}$ is the dust opacity, and $B_{v}\left(T_{\text {dust }}\right)$ is the Planck function for the dust temperature $T_{\text {dust }}$. We adopt $d=145 \mathrm{pc}$, which is the mean distance of the OB stars in the Upper Sco association (de Zeeuw et al. 1999). For consistency with Andrews et al. (2013), we adopt $\kappa_{v}=2.3 \mathrm{~cm}^{2} \mathrm{~g}^{-1}$ at $230 \mathrm{GHz}$ and assume $\kappa_{v}$ scales with frequency as $v^{\beta}$, where $\beta=0.4$. The dust temperature is estimated as $T_{\text {dust }}=25 \mathrm{~K} \times\left(L_{*} / L_{\odot}\right)^{0.25}$ (see Andrews et al. 2013). While a range of dust temperatures will be present in a disk, this formalism represents the characteristic dust temperature that describes the continuum emission.

Table 5 lists the derived dust masses. The uncertainties in the dust mass include the uncertainties in the measured flux densities and the distance to Upper Sco. The dust mass uncertainties do not include errors in the assumed dust opacity. However, the relative changes in the inferred dust masses may be more accurate to the extent that the dust properties are similar between disks.

\subsection{Dust Mass Versus Stellar Mass in Upper Sco}

Figure 3 shows the derived dust masses as a function of the stellar mass for the 20 stars in Upper Sco. The inferred dust masses of the sources detected with ALMA range over two orders of magnitude from $0.3 M_{\oplus}$ to $52 M_{\oplus}$, which represents $\sim 0.01 \%-1.7 \%$ of the stellar mass assuming a dust-to-gas ratio of 0.01 by mass. Considering both detections and upper limits, most disks have dust masses less than $1 M_{\oplus}$.

Disks around lower-mass stars tend to have lower dust masses than the disks around higher-mass stars. Eight of the nine stars with spectral type M3 or earlier $\left(M_{*}>0.26 M_{\odot}\right)$ were detected with ALMA. The one star not detected was one of the five stars that had lower sensitivity compared to the majority of the sample. By comparison, of the 11 M4 and M5 stars in the sample, only 5 were detected, even though all 11 stars had high-sensitivity data. Thus, the predominant number of stars 
Table 4

Measured Continuum Flux Densities at a Mean Frequency of $340.7 \mathrm{GHz}$

\begin{tabular}{|c|c|c|c|c|c|c|}
\hline Source & $\begin{array}{c}S_{\text {total }} \\
(\mathrm{mJy}) \\
(2)\end{array}$ & $\begin{array}{c}\Delta \alpha \\
(\operatorname{arcsec}) \\
(3)\end{array}$ & $\begin{array}{c}\Delta \delta \\
(\operatorname{arcsec}) \\
(4)\end{array}$ & $\begin{array}{c}\sigma \\
\left(\mathrm{mJy} \mathrm{beam}^{-1}\right) \\
(5)\end{array}$ & $\begin{array}{c}\theta_{\mathrm{b}} \\
(\operatorname{arcsec}) \\
(6)\end{array}$ & $\begin{array}{c}\text { P.A. } \\
\text { (deg) } \\
(7) \\
\end{array}$ \\
\hline [PBB2002]UScoJ155624.8 - 222555 & $0.28 \pm 0.18$ & $\ldots$ & $\ldots$ & 0.15 & $0.80 \times 0.48$ & -74 \\
\hline [PBB2002]UScoJ155706.4 - 220606 & $0.32 \pm 0.20$ & $\ldots$ & $\ldots$ & 0.20 & $0.91 \times 0.48$ & -73 \\
\hline [PBB2002]UScoJ155729.9 - 225843 & $-0.04 \pm 0.20$ & $\ldots$ & & 0.19 & $0.86 \times 0.48$ & -74 \\
\hline [PBB2002]UScoJ155829.8 - 231007 & $5.86 \pm 0.18$ & $0.10 \pm 0.11$ & $-0.01 \pm 0.11$ & 0.20 & $0.76 \times 0.48$ & -75 \\
\hline [PZ99]J160357.6 - 203105 & $4.30 \pm 0.39$ & $0.01 \pm 0.08$ & $0.06 \pm 0.08$ & 0.45 & $0.65 \times 0.48$ & -8 \\
\hline$[\mathrm{PZ99}] \mathrm{J} 160421.7-213028^{\mathrm{a}}$ & $218.76 \pm 0.81$ & $0.01 \pm 0.11$ & $-0.03 \pm 0.11$ & 0.19 & $0.76 \times 0.45$ & -74 \\
\hline [PBB2002]UScoJ160525.5 - 203539 & $1.53 \pm 0.20$ & $-0.09 \pm 0.19$ & $0.52 \pm 0.19$ & 0.19 & $0.99 \times 0.48$ & -72 \\
\hline [PBB2002]UScoJ160532.1 - 193315 & $0.25 \pm 0.20$ & $\ldots$ & $\ldots$ & 0.23 & $0.94 \times 0.48$ & -72 \\
\hline [PBB2002]UScoJ160600.6 - 195711 & $0.00 \pm 0.13$ & $\ldots$ & $\ldots$ & 0.13 & $0.68 \times 0.45$ & -76 \\
\hline ScoPMS31 & $4.08 \pm 0.52$ & $0.02 \pm 0.22$ & $0.50 \pm 0.22$ & 0.53 & $0.65 \times 0.49$ & -3 \\
\hline [PBB2002]UScoJ160622.8 - 201124 & $0.59 \pm 0.14$ & $0.09 \pm 0.19$ & $0.05 \pm 0.19$ & 0.13 & $0.70 \times 0.45$ & -75 \\
\hline [PBB2002]UScoJ160643.8 - 190805 & $1.11 \pm 0.42$ & $\ldots$ & $\ldots$ & 0.47 & $0.65 \times 0.48$ & -5 \\
\hline [PBB2002]UScoJ160702.1 - 201938 & $-0.09 \pm 0.20$ & $\ldots$ & $\ldots$ & 0.20 & $1.04 \times 0.48$ & -72 \\
\hline${ }^{[P B B 2002] U S c o J 160823.2 ~-~} 193001^{\mathrm{b}}$ & $43.19 \pm 0.81$ & $0.21 \pm 0.20$ & $0.29 \pm 0.21$ & 0.52 & $0.65 \times 0.50$ & -9 \\
\hline [PBB2002]UScoJ160827.5 - 194904 & $0.76 \pm 0.13$ & $0.01 \pm 0.15$ & $-0.03 \pm 0.15$ & 0.16 & $0.64 \times 0.45$ & -76 \\
\hline [PBB2002]UScoJ160900.0 - 190836 & $1.73 \pm 0.13$ & $0.04 \pm 0.12$ & $0.09 \pm 0.12$ & 0.14 & $0.66 \times 0.45$ & -76 \\
\hline [PBB2002]UScoJ160900.7 - 190852 & $47.28 \pm 0.91$ & $0.42 \pm 0.20$ & $-0.27 \pm 0.21$ & 0.62 & $0.65 \times 0.48$ & -4 \\
\hline [PBB2002]UScoJ160959.4 - 180009 & $0.67 \pm 0.18$ & $-0.19 \pm 0.26$ & $-0.13 \pm 0.26$ & 0.18 & $0.80 \times 0.48$ & -72 \\
\hline [PZ99]J161411.0 - 230536 & $4.77 \pm 0.14$ & $0.09 \pm 0.04$ & $-0.07 \pm 0.04$ & 0.16 & $0.70 \times 0.45$ & -76 \\
\hline [PBB2002]UScoJ161420.2 - 190648 & $40.69 \pm 0.22$ & $-0.12 \pm 0.20$ & $0.11 \pm 0.20$ & 0.15 & $0.77 \times 0.45$ & -73 \\
\hline
\end{tabular}

Notes.

Column 1: star name. Column 2: integrated flux density derived by fitting a point-source model to the $u v$ data, unless otherwise indicated. Columns 3 and 4: right ascension and declination offsets of the ALMA continuum source from the stellar position; ellipses indicate that the source was not detected with ALMA and the offsets were fixed at the stellar position during the model fitting. Column 5: rms noise in an image created with robust $=2$ and measured in an annulus between $4^{\prime \prime}$ and $5^{\prime \prime}$ centered on the stellar position. Column 6: FWHM synthesized beam size. Column 7: position angle of the beam measured east of north.

${ }^{a}$ Integrated flux density measured on an image with a circular aperture of 1 ". 5 radius.

${ }^{\mathrm{b}}$ Integrated flux density measured by fitting an elliptical Gaussian to the visibility data.

Table 5

Derived Dust Masses

\begin{tabular}{|c|c|}
\hline Source & $\log \left(M_{\text {dust }} / M_{\oplus}\right)$ \\
\hline [PBB2002]UScoJ155624.8 - 222555 & $<-0.34$ \\
\hline [PBB2002]UScoJ155706.4 - 220606 & $<-0.17$ \\
\hline [PBB2002]UScoJ155729.9 - 225843 & $<-0.40$ \\
\hline [PBB2002]UScoJ155829.8 - 231007 & $0.58 \pm 0.13$ \\
\hline [PZ99]J160357.6 - 203105 & $-0.01 \pm 0.14$ \\
\hline [PZ99]J160421.7 - 213028 & $1.72 \pm 0.13$ \\
\hline [PBB2002]UScoJ160525.5 - 203539 & $0.02 \pm 0.14$ \\
\hline [PBB2002]UScoJ160532.1 - 193315 & $<-0.12$ \\
\hline [PBB2002]UScoJ160600.6 - 195711 & $<-0.65$ \\
\hline ScoPMS31 & $0.00 \pm 0.14$ \\
\hline [PBB2002]UScoJ160622.8 - 201124 & $-0.38 \pm 0.17$ \\
\hline [PBB2002]UScoJ160643.8 - 190805 & $<-0.19$ \\
\hline [PBB2002]UScoJ160702.1 - 201938 & $<-0.33$ \\
\hline [PBB2002]UScoJ160823.2 - 193001 & $1.13 \pm 0.13$ \\
\hline [PBB2002]UScoJ160827.5 - 194904 & $-0.38 \pm 0.15$ \\
\hline [PBB2002]UScoJ160900.0 - 190836 & $0.05 \pm 0.13$ \\
\hline [PBB2002]UScoJ160900.7 - 190852 & $1.10 \pm 0.13$ \\
\hline [PBB2002]UScoJ160959.4 - 180009 & $-0.50 \pm 0.17$ \\
\hline [PZ99]J161411.0 - 230536 & $-0.16 \pm 0.13$ \\
\hline [PBB2002]UScoJ161420.2 - 190648 & $1.01 \pm 0.13$ \\
\hline
\end{tabular}

with non-detections are the late spectral types with lower stellar masses.

The significance of these apparent trends were evaluated using the correlation tests adapted for censored data sets (Isobe et al. 1986), as implemented in the ASURV software package (Lavalley et al. 1992). The Cox proportional hazard test, the

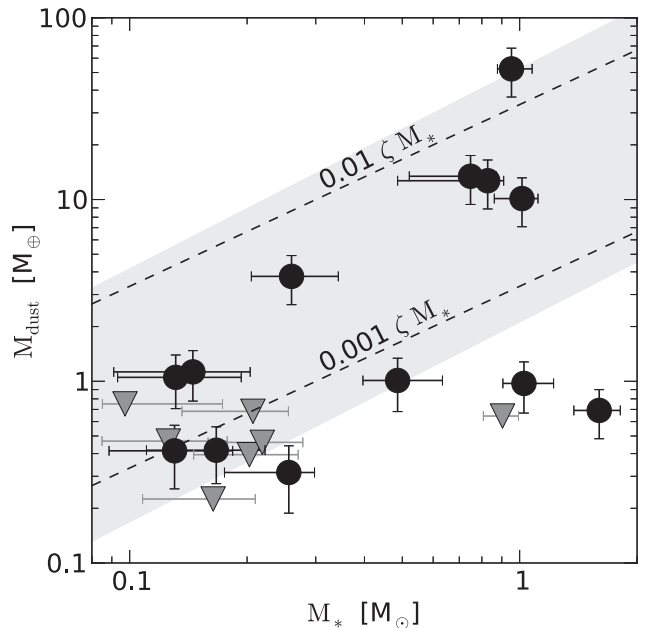

Figure 3. Dust mass as a function of stellar mass for the 20 stars in the Upper Sco sample. Sources with $880 \mu \mathrm{m}$ continuum detections are denoted by circles, and $3 \sigma$ upper limits to the $880 \mu \mathrm{m}$ detections are indicated by triangles. The dashed lines shows constant ratios of dust mass to stellar mass of $0.01 \zeta$ and $0.001 \zeta$, where $\zeta=0.01$ is the dust-to-gas ratio. The shaded region shows the correlation between dust mass and disk mass derived by Andrews et al. (2013) in Taurus, where the width of the region shows the 0.7 dex spread about the best-fit relation.

Kendall rank test, and the Spearman rank test in ASURV indicate that the probability of no correlation between dust mass and stellar mass is $0.017,0.044$, and 0.039 , respectively. We conclude that there is marginal evidence that the dust masses declines with stellar mass in the Upper Sco sample. 
Assuming a power-law relation is present between the dust masses and the stellar masses, the slope of the power law was derived using the Bayesian method described in Kelly (2007), which takes into account the measurement uncertainties, upper limits, and intrinsic scatter in the relationship. The derived relationship between the dust mass and the stellar mass is given by $\log \left(M_{\text {dust }} / M_{\oplus}\right)=(0.68 \pm 0.30)+(1.01 \pm 0.60) \log \left(M_{*} / M_{\odot}\right)$ with a spread of $0.76 \pm 0.24$ dex. Thus, the slope is consistent with a linear correlation between dust mass and stellar mass, but the uncertainties on the slope are such that no correlation is consistent with the data. The slope and spread for Upper Sco are consistent with that for disks in Taurus (slope $=1.1 \pm 0.4$; dispersion $=0.7 \pm 0.1 \mathrm{dex}$ ) found by Andrews et al. (2013), which is shown as the shaded region in Figure 3. In the following section, we present a more quantitative comparison between the Upper Sco and Taurus samples.

\section{COMPARISON BETWEEN UPPER SCO AND TAURUS}

The percentage of K- and M-type stars in Upper Sco that retain an optically thick inner disk is $\sim 19 \%$ (Carpenter et al. 2006) and may vary with stellar mass within this spectral-type range (Luhman \& Mamajek 2012). Given that $~ 80 \%$ of lowmass stars with an age of $\sim 1$ Myr contain a disk (Hernández et al. 2008), the average disk mass must be lower in Upper Sco compared to younger regions. However, the question remains if the dust masses stay relatively constant before dispersing rapidly or if there is a steady decline in the dust mass as it disperses. These different scenarios ultimately reflect the mass-loss rate in the disk and the mechanisms responsible for the disk dispersal. We aim to quantify this evolution by comparing submillimeter continuum observations toward stars of various ages that still retain optically thick disks.

In addition to the Upper Sco observations presented here (see also Mathews et al. 2012b), other star-forming regions that have been surveyed at submillimeter wavelengths include Taurus (Beckwith et al. 1990; Andrews \& Williams 2005; Andrews et al. 2013), $\rho$ Oph (André \& Montmerle 1994; Motte et al. 1998; Andrews \& Williams 2007b), IC 348 (Carpenter 2002; Lee et al. 2011), the Orion Nebula Cluster (Mann \& Williams 2009a, 2009b, 2010; Eisner et al. 2008) NGC 2024 (Eisner \& Carpenter 2003), MBM 12 (Itoh et al. 2003; Hogerheijde et al. 2002), Lupus (Nuernberger et al. 1997), Chamaeleon I (Henning et al. 1993), Serpens (Testi \& Sargent 1998), and $\sigma$ Orionis (Williams et al. 2013). Taurus is the one region that can be most readily compared with Upper Sco for a number of reasons. After decades of searching for members (see recent compilations by Rebull et al. 2010 and Luhman et al. 2010), the stellar census is likely nearly complete for sources with and without disks. A wealth of ancillary data, including spectral types, are available for most members so that a robust comparison can be made with Upper Sco over the same stellar mass range. Finally, the close proximity leads to improved sensitivity, as most disks around stars in Taurus that have spectral types earlier than M3 have been detected in the submillimeter continuum (Andrews et al. 2013).

\subsection{Relative Ages}

The age of Upper Sco is commonly assumed to be $~ 5 \mathrm{Myr}$ based on the kinematics of the B-type stars (Blaauw 1978) and placing association members in an $\mathrm{H}-\mathrm{R}$ diagram and inferring the age from evolutionary tracks (de Geus et al. 1989; Preibisch et al. 2002; Slesnick et al. 2008). More recently, Pecaut et al.
(2012) derived an age of $11 \pm 2$ Myr for Upper Sco based on the isochronal ages of the B-, A-, and G-type stars and the M supergiant Antares and the luminosities of the F-type stars. They also derive a lower limit of $10.5 \mathrm{Myr}$ ( $99 \%$ confidence) on the expansion age using radial velocities and Hipparcos parallaxes.

By comparison, the mean age of stars with disks in Taurus is $\sim 1-2 \mathrm{Myr}$, as inferred by placing stars in an H-R diagram (e.g., Kenyon \& Hartmann 1995; Hartmann 2001; Bertout et al. 2007; Andrews et al. 2013). Thus, Upper Sco appears older than Taurus. While one must be cautious of ages derived by different techniques, qualitative signatures also support the notion that Upper Sco is older. First, the natal molecular cloud has been dispersed as the visual extinction toward the association members is typically $A_{V}<2 \mathrm{mag}$. Also, the association lacks stars in the Class 0 and Class I phases that typify young starforming regions (e.g., Gutermuth et al. 2011). Finally, late-type stars in Upper Sco have surface-gravity photospheric spectral lines consistent with an older age relative to Taurus, and, in fact, has been used as a defining characteristic of membership (e.g., Slesnick et al. 2006). Thus, even though the age of Upper Sco may be uncertain by a factor of $\sim 2$, the association is almost certainly older than Taurus.

\subsection{Relative Dust Masses}

The comparison sample in Taurus consists of Class II sources compiled by Luhman et al. (2010, see also Rebull et al. 2010). The submillimeter flux densities for this sample are presented in Andrews et al. (2013), who used new and published submillimeter observations at multiple wavelengths to estimate the flux density at a wavelength of $890 \mu \mathrm{m}$. The Taurus submillimeter flux densities were extrapolated to the mean wavelength of the Upper Sco observations $(880 \mu \mathrm{m})$ by assuming the dust emission varies with frequency as $v^{2.4}$, which is the same frequency dependence adopted in Andrews et al. (2013).

It should be noted that the upper limits to the submillimeter flux densities for the Taurus and Upper Sco observations are not computed consistently. Upper limits in Taurus are generally reported as three times the rms noise of the observations, while the upper limits in Upper Sco derived here are given as three times the rms plus any positive measured flux density. Thus, the upper limits in Upper Sco are more conservative. Given the expectation that dust masses may be lower in the Upper Sco due to the older age, the different treatments of the upper limits will only weaken any differences in the two samples.

We analyzed the samples in two stellar mass ranges: 0.097-0.26 $M_{\odot}$ that encompasses the M3-M5 stars in Upper Sco, and 0.49-1.6 $M_{\odot}$ that encompasses the K2-M0.5 stars. The selection was done by stellar mass rather than spectral type since given the age differences between Upper Sco and Taurus, there is not a strict correspondence between spectral type and stellar mass. The stellar masses for the Taurus sample were derived from the effective temperatures and stellar luminosities in Andrews et al. (2013) using the interpolation procedure adopted for Upper Sco (see Section 2). The difference in $\log M_{*}$ derived here and those reported in Andrews et al. (2013) is 0.00 with a standard deviation of 0.05 dex.

The mass ranges of the two samples were motivated by three considerations. First, the observed Upper Sco sample contains no stars with spectral types between M0.5 and M3, which leads to a break in the stellar mass distribution. Second, Andrews et al. (2013) found a correlation between dust mass and stellar mass in that dust masses in disks in Taurus are $\sim 7 \times$ larger in the higher-mass bin than the lower-mass bin. Finally, the 


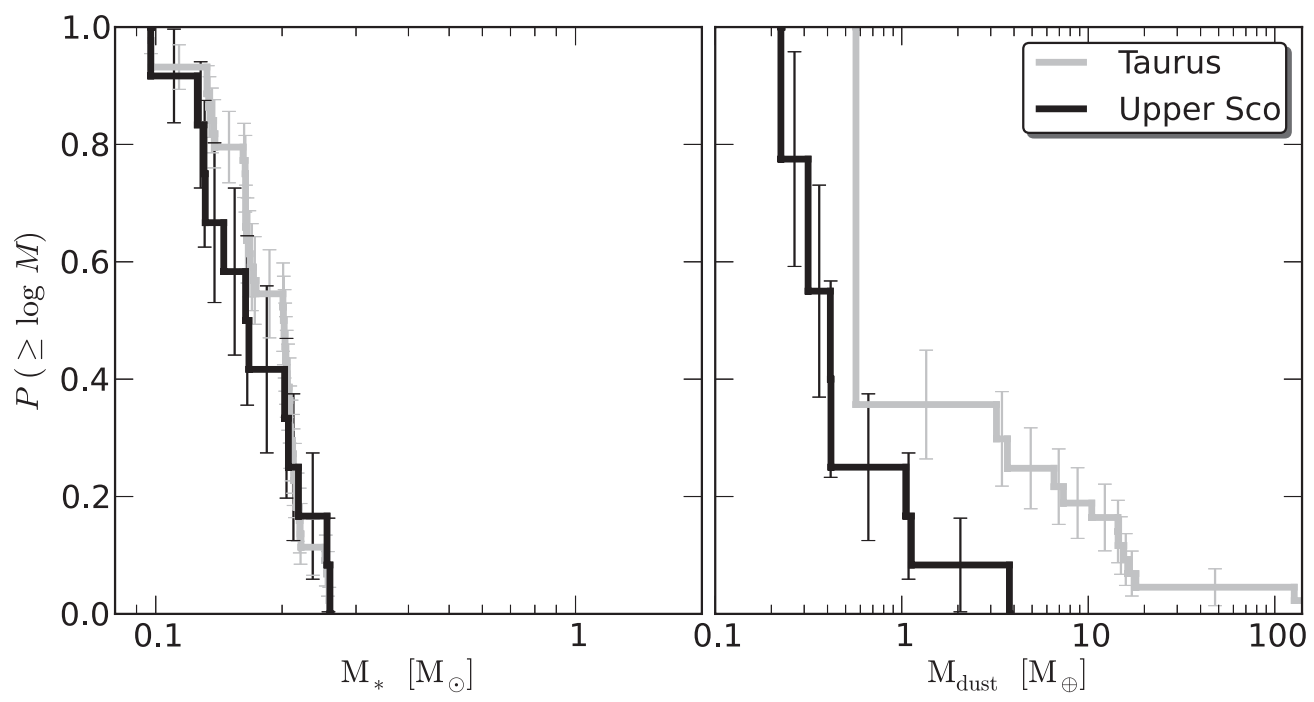

Figure 4. Left: cumulative distribution of the stellar masses for the Taurus (gray) and Upper Sco (black) samples for stellar masses between 0.097 and $0.26 M_{\odot}$. Right: cumulative distribution of the dust masses for the stellar samples shown in the left panel. Both the stellar masses and dust masses in Upper Sco and Taurus are consistent with being drawn from the same parent distribution.

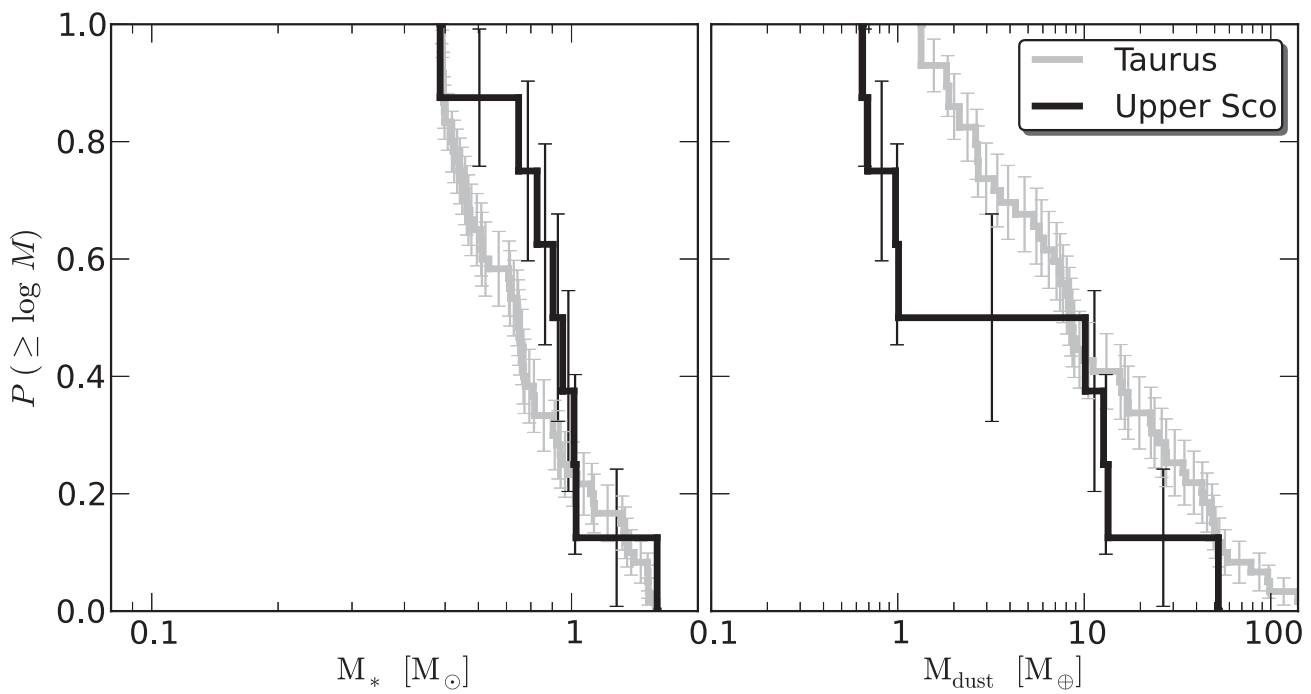

Figure 5. Same as in Figure 4, but for stellar masses between 0.49 and $1.6 M_{\odot}$. Both the stellar masses and dust masses in Upper Sco and Taurus are consistent with being drawn from the same parent distribution.

fraction of stars with optically thick disks as traced by infrared observations increases with decreasing stellar mass in Upper Sco (Carpenter et al. 2006; Luhman \& Mamajek 2012) and other regions (IC 348: Lada et al. 2006; NGC 2262: Dahm \& Hillenbrand 2007). The variations in the disk mass and disk lifetime with stellar mass suggests that the disk mass-loss rate may also vary with stellar mass.

Ideally, any comparison between Upper Sco and Taurus will consider the multiplicity of the stars since close companions can shorten the lifetime of disks (Jensen et al. 1996; Cieza et al. 2009; Harris et al. 2012; Kraus et al. 2012). However, multiplicity information is currently available for only 7 of the 20 Upper Sco stars observed with ALMA (Kraus et al. 2008). Therefore, we considered all of the stars in the Taurus sample that are within the appropriate stellar mass range. This could potentially bias the results if the remaining disks in Upper Sco are preferentially found around single stars.

We used the two-sample tests in the ASURV package to compare the Upper Sco and Taurus samples. The left panel in
Figure 4 compares the cumulative distribution of stellar masses in Taurus and Upper Sco for the lower-mass stars. For these stars, the median stellar mass in Taurus is $25 \%$ higher $\left(0.20 M_{\odot}\right.$ versus $0.16 M_{\odot}$ ) than in Upper Sco. However, the two-sample tests in the ASURV package indicate a probability of $0.37-0.96$ that the distribution of stellar masses are drawn from the same parent population. Similarly, for stars in the higher-mass bin (see left panel in Figure 5), the median stellar mass in the Taurus sample is $18 \%$ lower than in Upper Sco, but the probability that the stellar distributions are drawn from the same distribution is between 0.20 and 0.26 . Thus, there is no evidence for differences in the stellar mass distribution of Class II sources Taurus and Upper Sco for the two stellar mass ranges. Therefore, the dust mass distributions can be reliably compared between the two samples.

The right panel in Figure 5 shows the cumulative distributions of the dust masses around the lower-mass stars in the Taurus and Upper Sco samples as estimated using the Kaplan-Meier estimator to factor in upper limits. Formally, the mean dust 
mass in Upper Sco is $\left\langle\log \left(M_{\text {dust }} / M_{\oplus}\right)\right\rangle=-0.31 \pm 0.10$, which is lower than the mean mass in Taurus of $\left\langle\log \left(M_{\text {dust }} / M_{\oplus}\right)\right\rangle=$ $0.22 \pm 0.12$. However, only 7 of the 12 sources in Upper Sco were detected with ALMA, and only 12 of the 44 stars in Taurus. The Kaplan-Meier estimator requires that the censored data points be randomly distributed, which may not be valid for the lower-mass stars. Thus, the mean dust masses for the lowermass stars should be treated with caution. The ASURV twosample tests provide a robust comparison between the Upper Sco and Taurus samples that factor in the upper limits from the continuum observations and do not require that the censorship be random. These tests indicate the probability that the dust masses in the low-mass stars in Taurus and Upper Sco are drawn from the same parent population is between 0.064 and 0.086 . Thus, there is only marginal evidence that the lower-mass stars in the Upper Sco sample have lower disk masses than comparable stars in Taurus.

For the higher stellar mass bin, 7 of the 8 stars in Upper Sco and 48 of the 60 stars in Taurus were detected in the submillimeter continuum. The mean dust mass is $\left\langle\log \left(M_{\text {dust }} / M_{\oplus}\right)\right\rangle=$ $0.57 \pm 0.25$ for Upper Sco and $\left\langle\log \left(M_{\text {dust }} / M_{\oplus}\right)\right\rangle=1.01 \pm 0.08$ for Taurus. Thus, the dust mass distribution is skewed toward lower masses in Upper Sco compared to Taurus. However, the two-sample tests in ASURV indicate that the probability that the samples are drawn from the same parent population is between 0.03 and 0.21 . Therefore, the observed differences are not significant.

\section{CONCLUSIONS}

The results presented in Section 6 indicate that the distribution of dust masses between Taurus and Upper Sco are statistically indistinguishable given the present sample sizes. To place limits on the differences in the mean dust mass between Taurus and Upper Sco, we used the mean dust mass values from the Kaplan-Meier estimator presented in Section 6. We consider only the higher-mass stars $\left(0.49-1.6 M_{\odot}\right)$ given the preponderance of upper limits in Upper Sco and especially Taurus for the lower-mass stars. The change in the mean dust mass from Taurus to Upper Sco for the 0.49-1.6 $M_{\odot}$ stars is $\Delta \log M_{\text {dust }}=0.44 \pm 0.26$. Thus, the mean dust mass has declined by a factor of $\approx 2.8 \pm 1.6$; however, consistent with the analysis presented in Section 6, the uncertainties are such that no decline in the mean dust mass is consistent with the data. The $3 \sigma$ upper limit to the change in the mean $\log M_{\text {dust }}$ is $1.22 \mathrm{dex}$, and thus, formally, these data cannot exclude an order of magnitude change in the mean dust mass.

The reason why the constraints on the mean dust mass remain poor can be readily ascertained from Figure 3 . For the 0.49-1.6 $M_{\odot}$ stars, one-half have dust masses between $\sim 10$ and $50 M_{\oplus}$ and one-half have masses less than $1 M_{\oplus}$. The gap in the dust mass distribution within this stellar mass range implies that the median disk properties remain uncertain by an order of magnitude.

While the lower mean flux densities in Upper Sco relative to Taurus have been interpreted as a decrease in the dust masses, systematic differences in the dust composition or the grain size distribution can also lead to a decrease in the submillimeter flux density for a constant mass in solids. As an example, we computed the dust opacity by adopting the three most abundant species in the Pollack et al. (1994) dust composition and assuming that the size distribution of particles can be represented by a power law of $n(a) \propto a^{-3.5}$. Increasing the maximum particle radius to $1 \mathrm{~cm}$ from $1 \mathrm{~mm}$, but keeping the total mass in solids constant, would decrease the observed submillimeter flux density by a factor of 2.7 , which is consistent with the observed decrease in the flux density in Upper Sco relative to Taurus. In this scenario, the slope of the dust opacity between wavelengths of $1 \mathrm{~mm}$ and $3 \mathrm{~mm}$ will decrease to $\beta=0.66$ from $\beta=0.91$. While a systematic change of $\beta$ with stellar age has not been observed (Ricci et al. 2010a; Ubach et al. 2012), the uncertainties on the measurements for individual disks are typically $\Delta \beta \approx 0.2-0.4(1 \sigma)$. Thus we cannot exclude the possibility that the size distribution of particles is changing between Upper Sco and Taurus, but the overall mass of solids has remained the same. Sensitive, long-wavelength observations can help break the degeneracy between variations in grain growth and disk mass in accounting for the reduced submillimeter flux.

The sample for these observations was drawn from the Spitzer survey presented in Carpenter et al. (2006) for a subset of the known Upper Sco population. Since that time, not only has the census of Upper Sco members been refined, but the all-sky WISE survey between 3.5 and $22 \mu \mathrm{m}$ has also been completed, which can be used to assess the presence of a disk in all association members. Such a census has already by completed (Rizzuto et al. 2012; Luhman \& Mamajek 2012), and there are over 200 stars and brown dwarfs over all spectral types in Upper Sco that have infrared excess characteristics of a disk, including primordial and debris disks. Future observations of this large sample with ALMA will probe the tentative correlations identified in this paper.

We thank Crystal Brogan and Steve Myers for their assistance in the data reduction. The National Radio Astronomy Observatory is a facility of the National Science Foundation operated under cooperative agreement by Associated Universities, Inc. This paper makes use of the following ALMA data: ADS/JAO.ALMA\#2011.0.00526.S. ALMA is a partnership of ESO (representing its member states), NSF (USA), and NINS (Japan), together with NRC (Canada) and NSC and ASIAA (Taiwan), in cooperation with the Republic of Chile. The Joint ALMA Observatory is operated by ESO, AUI/NRAO, and NAOJ. A.I. and J.M.C. acknowledge support from NSF awards AST-1109334 and AST-1140063. This publication makes use of data products from the Two Micron All Sky Survey, which is a joint project of the University of Massachusetts and the Infrared Processing and Analysis Center/California Institute of Technology, funded by the National Aeronautics and Space Administration and the National Science Foundation.

\section{REFERENCES}

André, P., \& Montmerle, T. 1994, ApJ, 420, 837

Andrews, S. M., Rosenfeld, K. A., Kraus, A. L., \& Wilner, D. J. 2013, ApJ, 771,129

Andrews, S. M., \& Williams, J. P. 2005, ApJ, 631, 1134

Andrews, S. M., \& Williams, J. P. 2007a, ApJ, 659, 705

Andrews, S. M., \& Williams, J. P. 2007b, ApJ, 671, 1800

Andrews, S. M., Wilner, D. J., Hughes, A. M., Qi, C., \& Dullemond, C. P. 2009, ApJ, 700, 1502

Andrews, S. M., Wilner, D. J., Hughes, A. M., Qi, C., \& Dullemond, C. P. 2010, ApJ, 723, 1241

Beckwith, S. V. W., Sargent, A. I., Chini, R. S., \& Gs̈ten, R. 1990, AJ, 99, 924 Bertout, C., Siess, L., \& Cabrit, S. 2007, A\&A, 473, L21

Blaauw, A. 1978, in Problems of Physics and Evolution of the Universe, ed. L. V. Mirzoyan (Yerevan: Armenian Academy of Sciences), 101

Cardelli, J. A., Clayton, G. C., \& Mathis, J. S. 1989, ApJ, 345, 245

Carpenter, J. M. 2002, AJ, 124, 1593

Carpenter, J. M., Mamajek, E. E., Hillenbrand, L. A., \& Meyer, M. R. 2006, ApJL, 651, L49

Cieza, L. A., Padgett, D. L., Allen, L. E., et al. 2009, ApJL, 696, L84 
Cutri, R. M., Skrutskie, M. F., van Dyk, S., et al. 2003, yCat, 2246, 0

Dahm, S. E., \& Carpenter, J. M. 2009, AJ, 137, 4024

Dahm, S. E., \& Hillenbrand, L. A. 2007, AJ, 133, 2072

de Geus, E. J., de Zeeuw, P. T., \& Lub, J. 1989, A\&A, 216, 44

de Zeeuw, P. T., Hoogerwerf, R., de Bruijne, J. H. J., Brown, A. G. A., \& Blaauw, A. 1999, AJ, 117,354

Dutrey, A., Guilloteau, S., Duvert, G., et al. 1996, A\&A, 309, 493

Eisner, J. A., \& Carpenter, J. M. 2003, ApJ, 598, 1341

Eisner, J. A., Plambeck, R. L., Carpenter, J. M., et al. 2008, ApJ, 683, 304

Guilloteau, S., Dutrey, A., Piétu, V., \& Boehler, Y. 2011, A\&A, 529, 105

Gutermuth, R. A., Pipher, J. L., Megeath, S. T., et al. 2011, ApJ, 739, 84

Haisch, K. E., Jr., Lada, E. A., \& Lada, C. J. 2001, ApJL, 553, L153

Harris, R. J., Andrews, S. M., Wilner, D. J., \& Kraus, A. L. 2012, ApJ, 751, 115

Hartmann, L. 2001, AJ, 121, 1030

Henning, T., Pfau, W., Zinnecker, H., \& Prusti, T. 1993, A\&A, 276, 129

Hernández, J., Calvet, N., Hartmann, L., et al. 2005, AJ, 129, 856

Hernández, J., Hartmann, L., Calvet, N., et al. 2008, ApJ, 686, 1195

Hogerheijde, M. R., Jawardhana, R., Johnstone, D., Blake, G. A., \& Kessler, J. E. 2002, AJ, 124, 3387

Isella, A., Carpenter, J. M., \& Sargent, A. I. 2009, ApJ, 701, 260

Isobe, T., Feigelson, E. D., \& Nelson, P. I. 1986, ApJ, 306, 490

Itoh, Y., Sugitani, K., Fukuda, N., et al. 2003, ApJL, 586, L141

Jensen, E. L. N., Mathieu, R. D., \& Fuller, G. A. 1996, ApJ, 458, 312

Kelly, B. C. 2007, ApJ, 665, 1489

Kenyon, S. J., \& Hartmann, L. 1995, ApJS, 101, 117

Kitamura, Y., Momose, M., Yokogawa, S., et al. 2002, ApJ, 581, 357

Kraus, A. L., Ireland, M. J., Hillenbrand, L. A., \& Martinache, F. 2012, ApJ, 745,19

Kraus, A. L., Ireland, M. J., Martinache, F., \& Lloyd, J. P. 2008, ApJ, 679,762

Kwon, W., Looney, L. W., \& Mundy, L. G. 2011, ApJ, 741, 3

Lada, C. J., Muench, A. A., Luhman, K. L., et al. 2006, AJ, 131, 1574

Lada, C. J., \& Wilking, B. A. 1984, ApJ, 287, 610

Lavalley, M., Isobe, T., \& Feigelson, E. 1992, in ASP Conf. Ser. 25, Astronomical Data Analysis Software and Systems I, ed. D. M. Worrall, C. Biemesderfer, \& J. Barnes (San Francisco, CA: ASP), 245
Lee, N., Williams, J. P., \& Cieza, L. A. 2011, ApJ, 736, 135

Luhman, K. L., Allen, P. R., Espaillat, C., Hartmann, L., \& Calvet, N. 2010, ApJS, 186, 111

Luhman, K. L., \& Mamajek, E. E. 2012, ApJ, 758, 31

Mamajek, E. E., Meyer, M. R., Hinz, P. M., et al. 2004, ApJ, 612, 496

Mann, R. K., \& Williams, J. P. 2009a, ApJL, 694, L36

Mann, R. K., \& Williams, J. P. 2009b, ApJL, 699, L55

Mann, R. K., \& Williams, J. P. 2010, ApJ, 725, 430

Mathews, G. S., Williams, J. P., \& Ménard, F. 2012a, ApJ, 753, 59

Mathews, G. S., Williams, J. P., Ménard, F., et al. 2012b, ApJ, 745, 23

Mayama, S., Hashimoto, J., Muto, T., et al. 2012, ApJL, 760, L26

Motte, F., André, P., \& Neri, R. 1998, A\&A, 336, 150

Nuernberger, D., Chini, R., \& Zinnecker, H. 1997, A\&A, 324, 1036

Pecaut, M. J., \& Mamajek, E. E. 2013, ApJS, 208, 9

Pecaut, M. J., Mamajek, E. E., \& Bubar, E. J. 2012, ApJ, 765, 154

Pollack, J. B., Hollenbach, D., Beckwith, S., et al. 1994, ApJ, 421, 615

Preibisch, T., Brown, A. G. A., Bridges, T., Guenther, E., \& Zinnecker, H. 2002, AJ, 124, 404

Rebull, L. M., Padgett, D. L., McCabe, C.-E., et al. 2010, ApJS, 186, 259

Ricci, L., Testi, L., Natta, A., \& Brooks, K. J. 2010a, A\&A, 521, 66

Ricci, L., Testi, L., Natta, A., et al. 2010b, A\&A, 512, 15

Rizzuto, A. C., Ireland, M. J., \& Zucker, D. B. 2012, MNRAS, 421, L97

Roeser, S., Demleitner, M., \& Schilbach, E. 2010, AJ, 139, 2240

Schaefer, G. H., Dutrey, A., Guilloteau, S., Simon, M., \& White, R. J. 2009, ApJ, 701, 698

Siess, L., Dufour, E., \& Forestini, M. 2000, A\&A, 358, 593

Simon, M., Dutrey, A., \& Guilloteau, S. 2000, ApJ, 545, 1034

Skrutskie, M. F., Cutri, R. M., Stiening, R., et al. 2006, AJ, 131, 1163

Slesnick, C. L., Carpenter, J. M., Hillenbrand, L. A., \& Mamajek, E. E. 2006, AJ, 132,2665

Slesnick, C. L., Hillenbrand, L. A., \& Carpenter, J. M. 2008, ApJ, 688, 377

Testi, L., \& Sargent, A. I. 1998, ApJL, 508, L91

The DENIS Consortium. 2005, yCat, 2263, 0

Ubach, C., Maddison, S. T., Wright, C. M., et al. 2012, MNRAS, 425, 3137

Williams, J. P., Cieza, L. A., Andrews, S. M., et al. 2013, MNRAS, 435, 1671

Zhang, K., Isella, A., Carpenter, J. M., \& Blake, G. A. 2014, ApJ, submitted 